\title{
Space Station Reboost with Electrodynamic Tethers
}

\author{
Irwin E. Vas," Thomas J. Kelly, ${ }^{\dagger}$ and Ethan A. Scarl* \\ The Boeing Company, Huntsville, Alabama 35824-6402
}

\begin{abstract}
This paper presents the results of a study of an electrodynamic tether system to reboost the International Space Station (ISS). One recommendation is to use a partially bare tether for electron collection. Locations are microgravity environment ther system is to be attached at the space station. The effects of the tether system on the periods and, if deployed from actually be beneficlal, because the system can neutrallze aerodrag during quiescent acceleration con relatively short tether system, $7 \mathrm{~km}$ ang opches to tether deployment and retrleval are discussed. It is shown that a over a billion dollars during 10 km long, operating at a power level of $5 \mathrm{~kW}$ could provide cumulative savings of number of flights thating a 10-year period ending in 2012. This savings is the direct result of a reduction in the higher tether usage. In addition to econome required to deliver propellant for reboost, with larger cost savings for system that could ensure ISS survival in the event of an (otherwise) catastrophic delay in propellant delivery.
\end{abstract}

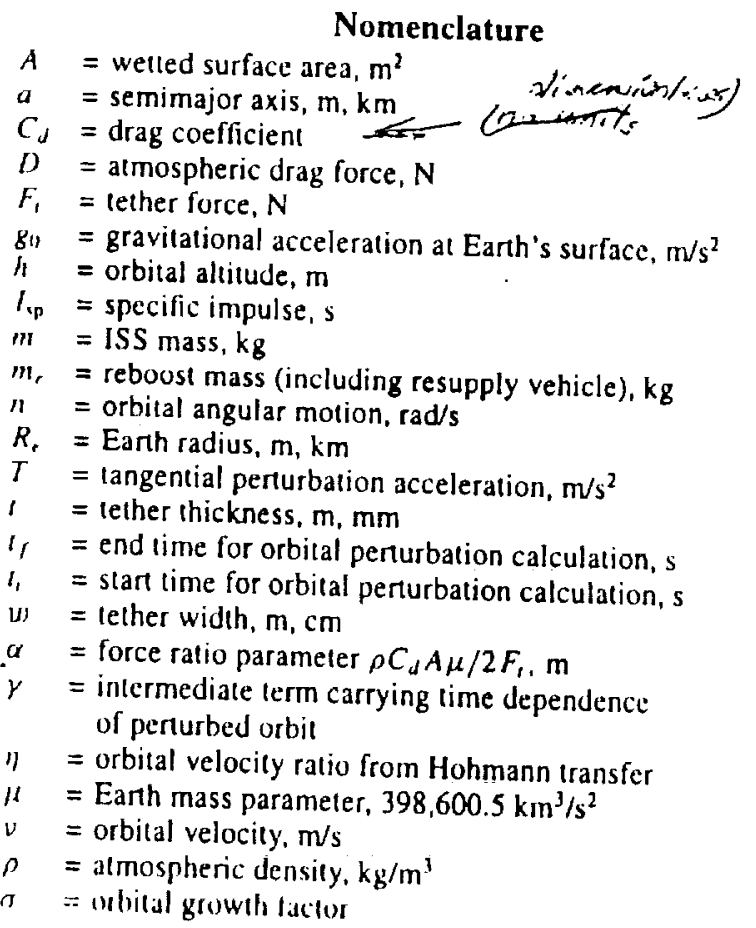

\section{Nomenclature}

\section{Introduction}

T HERE has been a renewed focus on reboost of the Internalional Space Station (ISS), caused in part by delays in the delivery of the Russian Service Module and the use of the Progress spacecralt. The current approach to reboost the ISS is by regular flights of the Russian Progress $M$ to replenish propellant. Several other reboos propellant carriers/reboost vehicles have been proposed such as the Progress M2, Propulsion Control Module, Interim Conlrol Module. and at variation of the Inertial Upper Stage. The Progress $M$ is the unly existing vehicle that performs this task. All other prospective vehicles must undergo major modification or have yet to be designed

$$
\text { (C) Boeing } 2000 \text { All rights therved. }
$$

Received 21 September 1998; revision received $20 \mathrm{March} 1999$; accepted for publication 7 September 1999

$$
\begin{aligned}
& \text { is:Te. Lob tille]. Space and Communications Group, } 499 \text { Boeing Boulevard. } \\
& \text { Associate Fellow AIAA. } \\
& \text { 'Gob title]. Space and Communications Group, } 499 \text { Bocing Boulevard. } \\
& \text { Mernber AlAA. }
\end{aligned}
$$

and built. A different approach, presented in this paper, provides reboost by a propellantless method.

There has been extensive work carried out with tethers in space. Most of this work has been documented in conference proceedings up to 1995.' The first demonstration of a nonconducting tether look place in 1967 with Gemini II in low Earth orbit illustrating gravily gradient stabilization. Most of the flight demonstrations, however, have taken place in this decade for both nonconducting and conducting tethers. Electrodynamic tethers have been demonstrated in space on a number of missions. The Tethered Satellite System (TSS) was an orbiter-based system, which deployed to a length of $19 \mathrm{~km}$ and generated approximately $2 \mathrm{~kW}$ of electrical power. The Plasma Motor Generator was flown as a secondary payload on a Delta II which deployed to a length of $0.5 \mathrm{~km}$ and successfully demon. strated the principles of electrodynamic tether reboost. The Small Expendable Deployer System flew twice as a secondary payload on Delta II launches, which demonstrated hollow cathode current collection limits from $200-900 \mathrm{~km}$. In addition, an electrodynamic lether propulsion for upper-stage applications is planned for development as part of the Advanced Space Transportation Program.

Stability of the ISS' low Earth orbit will be reduced as a trade. off for improvement in economy of access. Low-altitude orbits are economically viable because of the greater payload that each supply night can deliver, even at the cost of aerodynamic drag so high that the orbit is vulnerable to collapse if not reboosted every few minulhs. With frequent reboost thus designed to be an essential part of the ISS' life cycle, the practicality and economics of alternative reboos! methods need to be evaluated carefully.

An electrodynamic tether is the only reboost method capable of using solar energy as an alternative to consuming propellant. It exploits the fact that although this near-Earth environment burdens us with signilicant aerodynamic drag it also provides us wilh bolh a magnetic field and a conduclive medium. The thin environmental plasma is capable of closing a tether circuit without transinitting the resulting electrodynamic deceleration to the tether or its altached platforin. In fact, the environmental conductivity is large enough that the tether must be insulated along any portion intended to generate voltage or thrust, so as to keep the surrounding plasma from shorting
it oul.

Because it is easier to collect electrons at the far end of a tether and easier to reemit them from an electron gun (a plasma contactor) on the platform, electrodynamic tethers are usually operated with their negative end at the platform. They are directed to nadir for reboost or lo zenith for power generation. This mechanism is forluilous, but the driving current is limited to the excess capacity of the station's plasma contactor, assumed now to be about 5 A. Powering the tether with $5-10 \mathrm{~kW}$ will therefore require approximately $1-2 \mathrm{kV}$, which is roughly the voltage limit of what is presumed acceptable at the 
station surface for even an insulated connection. To achieve this voltage, the tether length would need to be between 5 and $15 \mathrm{~km}$ long.

This paper will review the principles of using an electrodynamic tether to counteract aerodynamic drag. We then present several associated practical design issues with altemative approaches to their resolution. The mechanics of flight and reboost in a quasi-stable orbit are presented in the next section. Deployment issues are then discussed, including novel approaches, followed by consideration of requirements for power management and physical attachment. The following section considers alternative policies for reboost scheduling. We conclude with discussion of a lether's physical and operational impacts on the ISS and a calculation of net cost savings.

\section{ISS Drag and Reboost Propellant Needs}

The results presented in this paper are derived from a study? conducted in 1996, which assumed that the first element of the ISS would be in orbit in late 1997. Even though there is a delay in the flight assembly sequence, the trends illustrated here remain valid. With the initial flight having taken place in 1998, full ISS assembly is expected in 2005 .

This study uses results from Design and Analysis Cycle 4 (DAC \#4) as a baseline for the altitude profile and reboost propellant needs of the ISS. ${ }^{3}$ During the buildup phase and subsequent years of operation, the planned altitude of the ISS is shown in Fig. 1. The altitude profile of the ISS is subjected to a number of competing performance requirements, such as the microgravity quality, resupply limitation, and the demand for 180 days per year of acceptable microgravity. Satisfying these requirements is complicated by the variations in atmospheric density, which exhibits daytime highs and nightime lows, as well as larger but slower variations driven by the 11-year solar cycle. In Fig. I the steep negative slopes are the result of orbital decay caused by aerodynamic drag, whereas the sharply positive slopes are the result of orbit raising propellant reboost maneuvers. Nole that these reboost maneuvers do not occur during quiescent microgravity periods. The propellant required to keep the stalion in its planned orbit is approximately 135 metric tons over the assembly phase from 1998 to 2005 , and the 10-year operational phase from 2005 to 2014 . The distribution of propellant over this period of time is shown in Fig. 2. An agreement made between the United States and Russia in June 1996 stated that the United States was responsible for $71 \%$ of the total propellant demand. In addition to maintaining orbit altitude, propellant is required by the reaction control system to periodically off-load the momentum accumulated in the attitude control system to prevent its saturation.

The aerodynamic drag force $D$ exerted on the ISS is directed opposite its orbital velocity vector according to the familiar relalionship

$$
D=\frac{1}{2} \rho v^{2} C_{d} A
$$

For the range of altitudes shown in Fig. 1, atmospheric density varies between $10^{-13}$ and $10^{-11} \mathrm{~kg} / \mathrm{m} .{ }^{3}$ The circular orbital velocity of the ISS is described by

$$
v=\sqrt{\mu /\left(R_{e}+h\right)}
$$

The welled surface area was computed from

$$
A=m / \beta C_{d}
$$

The DAC \#4 data for ballistic coefficient $\beta$ and ISS mass $m$ were used. The drag coefficient was set to $C_{d}=2.35$, which corresponds to a worst case of low-density free molecular flow. Note that changes in $A$ are small following assembly completion. This results in the orbit-averaged aerodynamic drag profile illustrated in Fig. 3.

\section{Tether Environment and Electron Collection}

\section{Tether Thrust}

The thrust produced by a conducting tether, driven by a given power level, depends upon the magnetic field, the orbital velocity, and the tether current. Whereas velocity is predictable and constant for a circular orbit, the Earth's magnetic field can vary by a factor of two. Furthermore, the current depends upon the combination of driving voltage and plasma electron density, the latter affecting the conductivity of the current's return path. The local electron density depends upon the effects of solar radiation; it therefore depends upon the phase of the solar cycle and whether the orbital segment is in sunlight and can easily change by an order of magnitude over a single orbit. Figure 4 shows the dramatic variation of electron density as the 90 -min orbit moves between day and night environments. The strongest fluctuations are caused by variations in exposure to

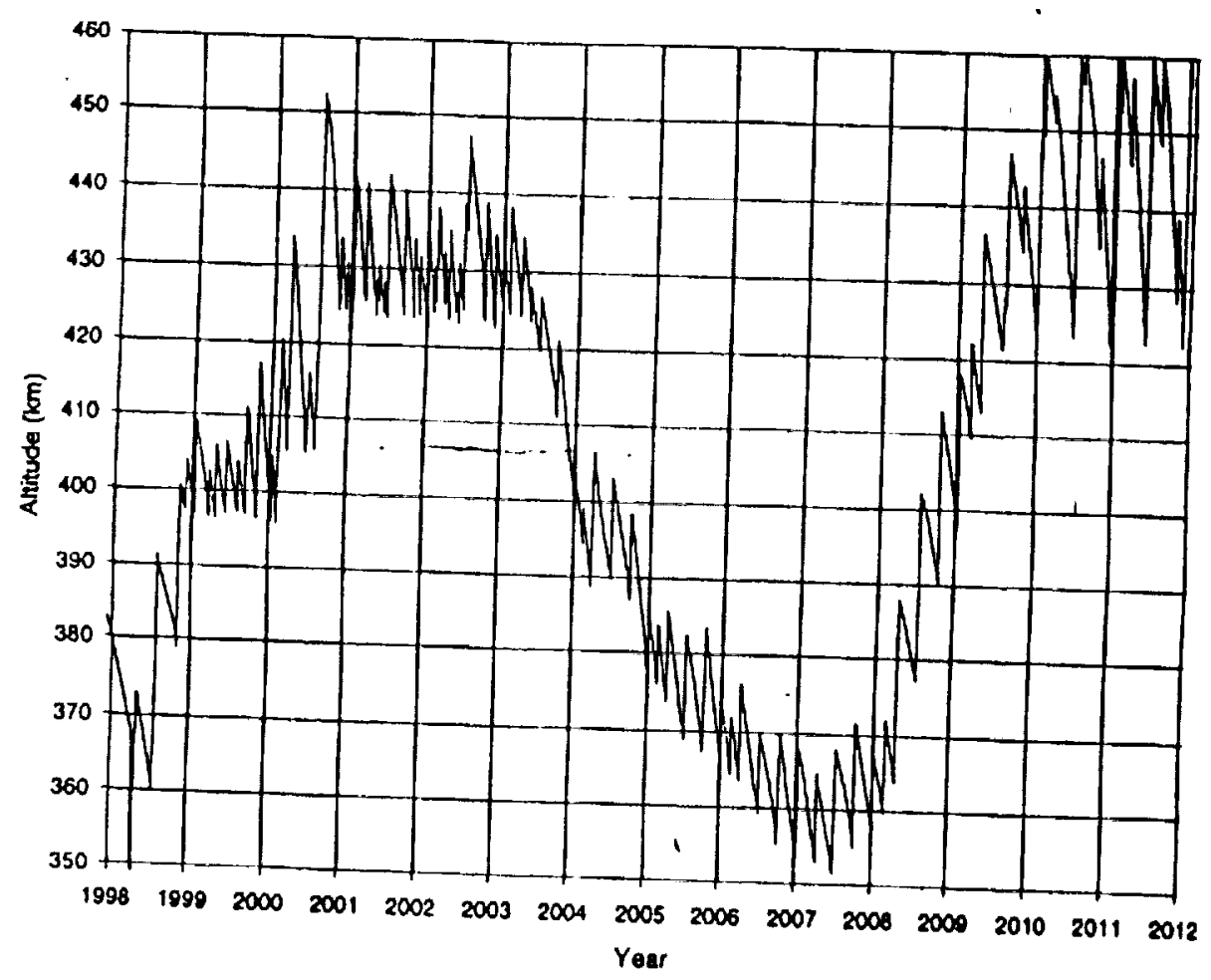

Fig. I Recommended space station altitude. 


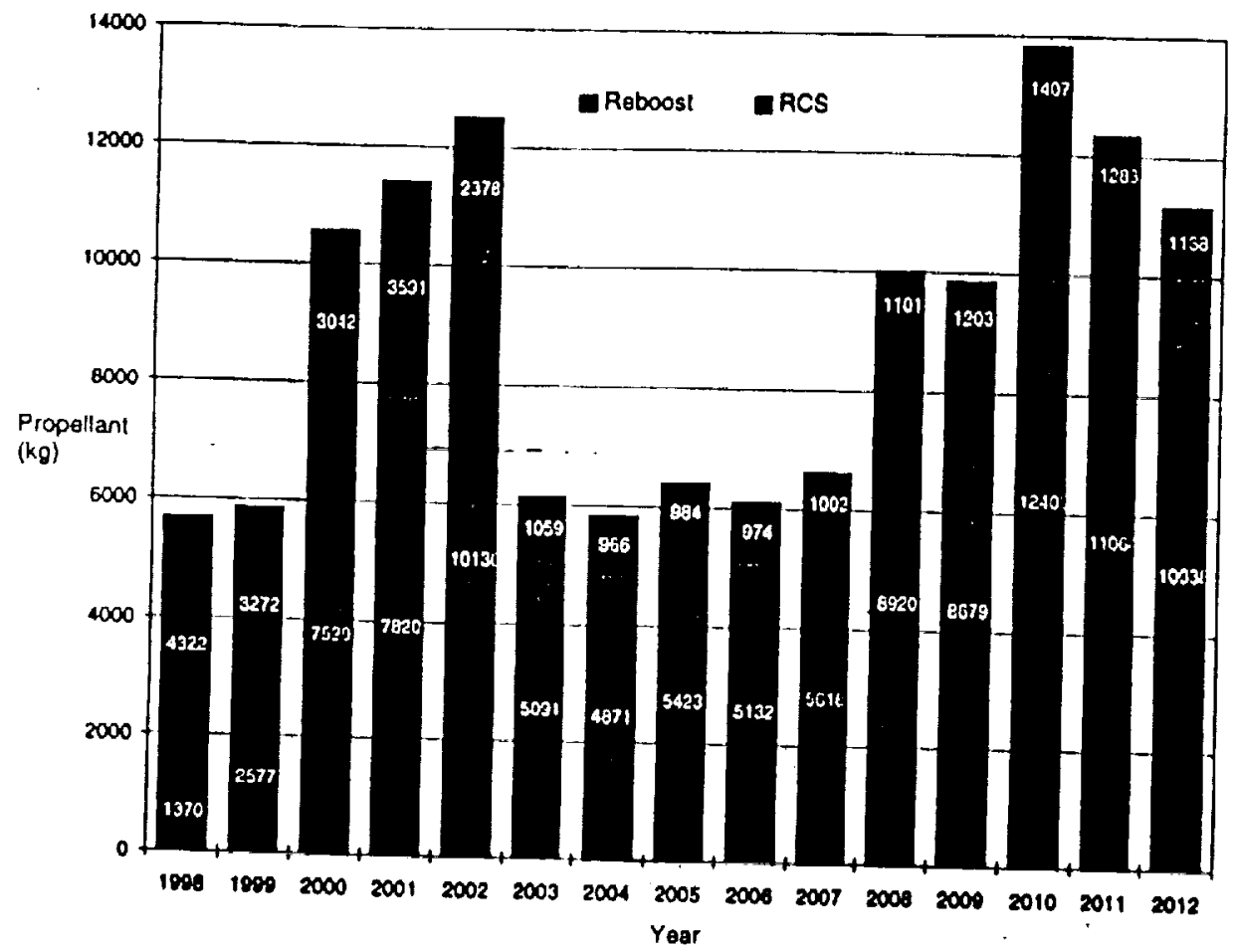

Fig. 2 ISS annual propellant requlrement.

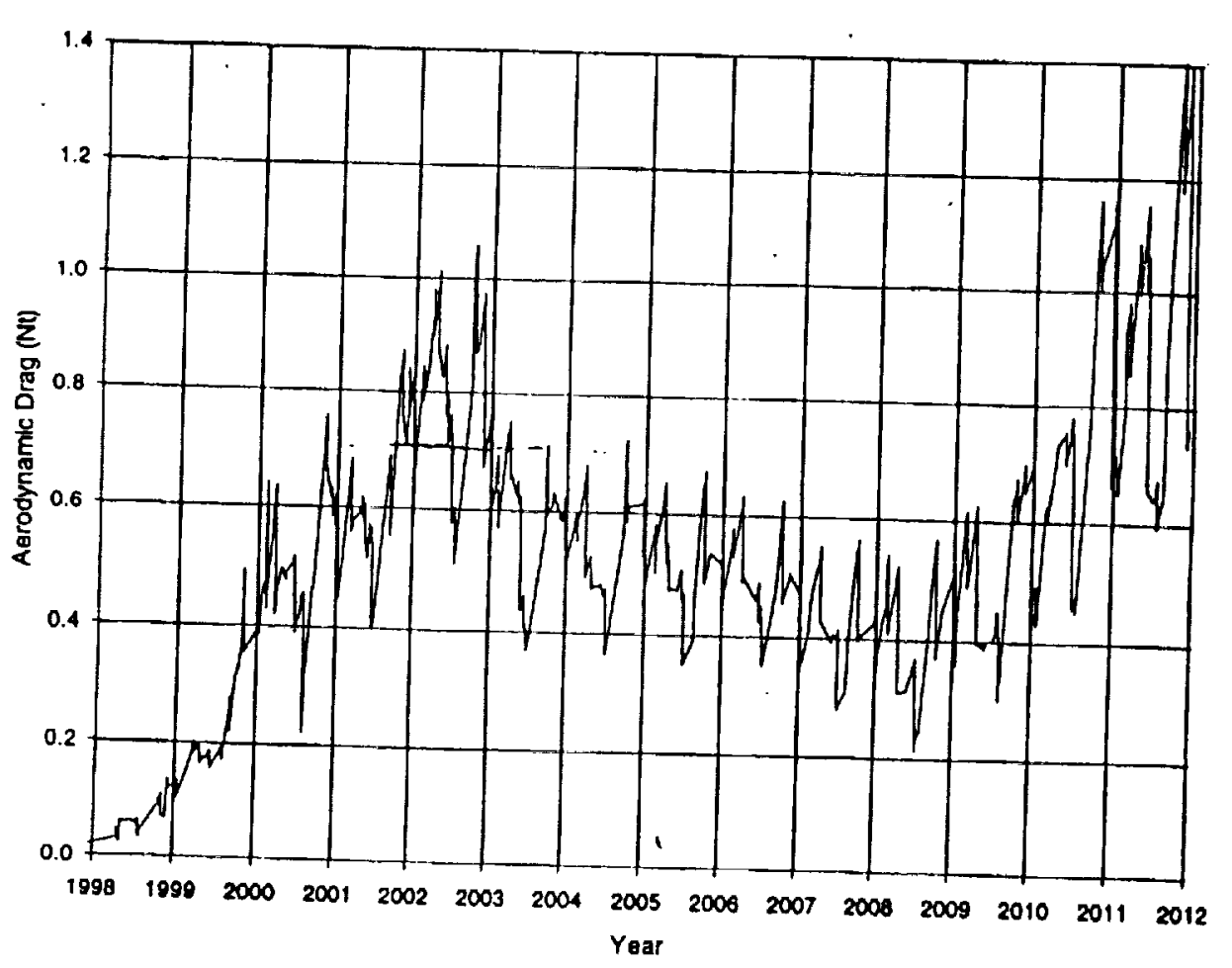

Fig. 3 Space station orbit-averaged aerodynamic drag.

sunlight and the solar wind, with other periodicities caused by the changing regions of the magnetusphere intercepted by the orbit. The voltage induced by the orbital motion, which affects the voltage that a power supply must impose upon the tether to achieve a given current, follows a similar curve, Fig. 5, and reflects variations in the field magnitude and the angle between field and orbit plane. These factors cause variations in tether efficiency, effective tether length (with a bare-tether electron collector), and the driving voltage required to compensate changes in induced voltage and plasma conductivity. Under reasonable assumptions, the resulting orbital variations in lether thrust are shown in Fig. 6 for a $7-\mathrm{km}$ tether driven with $5 \mathrm{~kW}$ and are seen to vary between 0.22 and $0.53 \mathrm{~N}$.

\section{Electrodynamic Tether Drag}

Determination of the tether's own aerodynamic drag force only differs from the ISS drag-force calculation in that the values used for the wetted surface area and drag coefficient are different. The projected area of a nadir directed tether of rectangular cross section will vary as it rotates and/or twists. For a tether of thickness $t=0.6 \mathrm{~mm}$ and width $w=1.1 \mathrm{~cm}$, the wetted surface area per $\mathrm{km}$ is at most

$$
1000(w+t) 2 / \pi \approx 6.8 \mathrm{~m}^{2} / \mathrm{km}
$$

A tether drag coefficient of 2.2 was used, which is a nominal value for a plate-like object in low-densily free molecular flow. As 


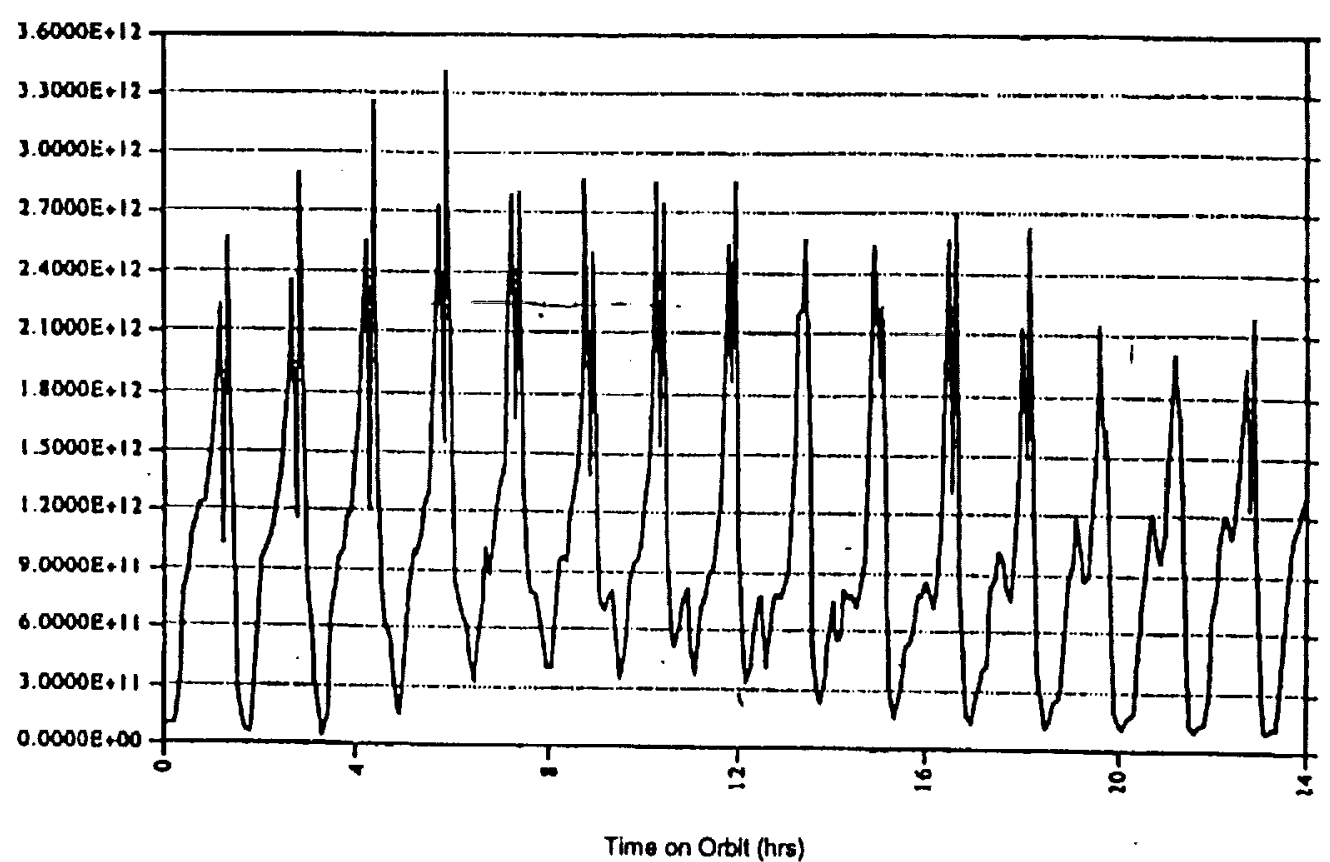

Fig. 4 Eleciron density variatlons.

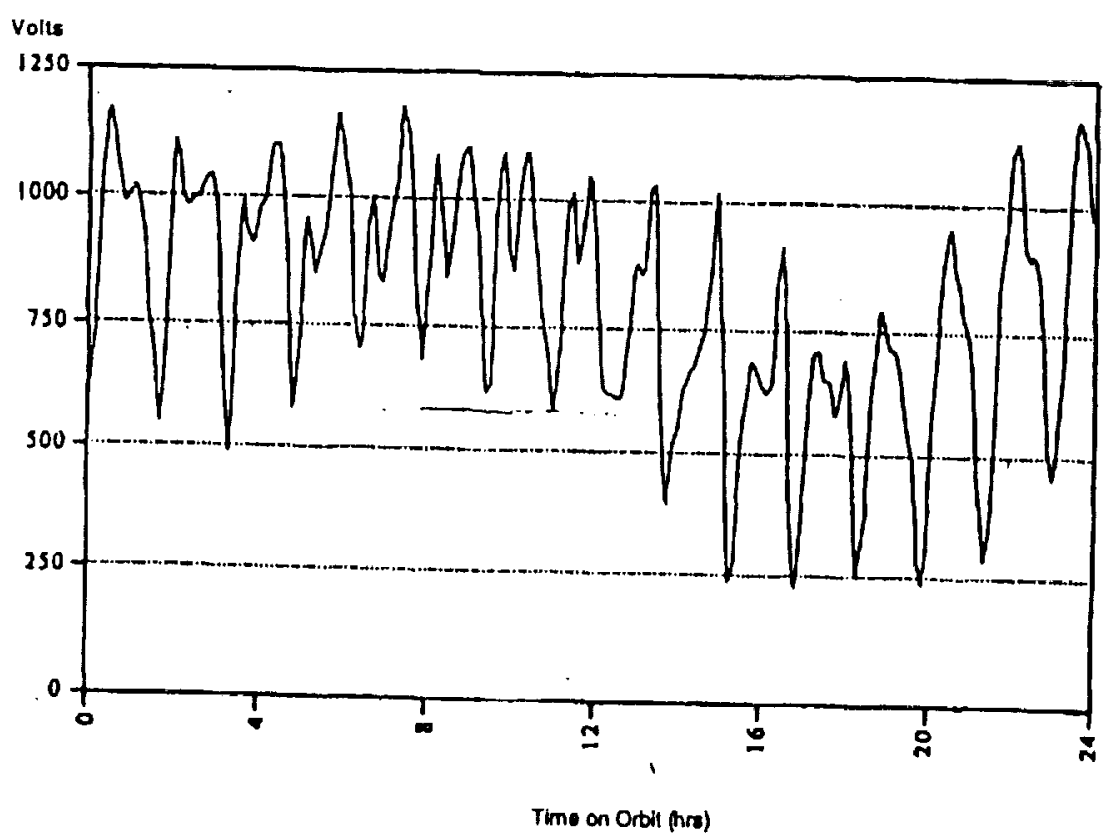

Fig. 5 Motional voltage variations.

illustrated in Fig. 7, the tolal tether drag force was found to bc approximately $6 \%$ of the ISS drag-force value.

\section{Bare Tether for Electron Collection}

Any metallic surface at the end of the tether will serve to collect clectrons. A long thin collector, such as an uninsulated extension of an insulated conducting tether, is a more efficient at electron collection than a sphere of the same surface area. This bare-tether collector has the interesting property that its aclive collecting length naturally varies to compensate the significant changes in plasmil clectron density that occur as the platform moves through different regions of the magnetosphere, especially between the day and night sides of the Eanh. This process is illustrated schematically in Fig. 8. in which the continuous behavior of the tether is approximated by a series of discrete incremental segments of length $\Delta L$. Each $\Delta L$ has resistance $\Delta R$. Most of these lie in the insulated por- tion $L_{1}$, from which current cannot leak. Along the uninsulated end of the tether, the continuous distribution of leakage path is shown as a discrete set of diodes to indicate that current can leave but not enter the tether (i.e., electrons can be captured but not emitted) The $y$ of a diode corresponds to its connection to the plasma. If the forward conductivity of the diode in the first segment $\Delta L$ of uninsulated tether (the first diode path $I_{1}$ ) is sufficiently great, most of the current $I$ follows this path so that $I \cong I_{1}$, and the remaining currents $\left(I_{n}\right.$ for $\left.n>1\right)$ are all nearly zero. If the plasma conductivity of the first uninsulated segment is less than perfect, however, the next $\Delta L$ will have some voltage drop $\Delta V$ across it, and part of the remaining tether current $\left(I-I_{1}\right)$ will flow as $I_{2}$. At night, or under other conditions of lowered free electron density in the plasma. these diode conductivities will be lower. More of them will then be involved in conducting the full current $I$, and the positive voltage will extend further along the bare tether. Thus, the bare tether 
Thrust (N)

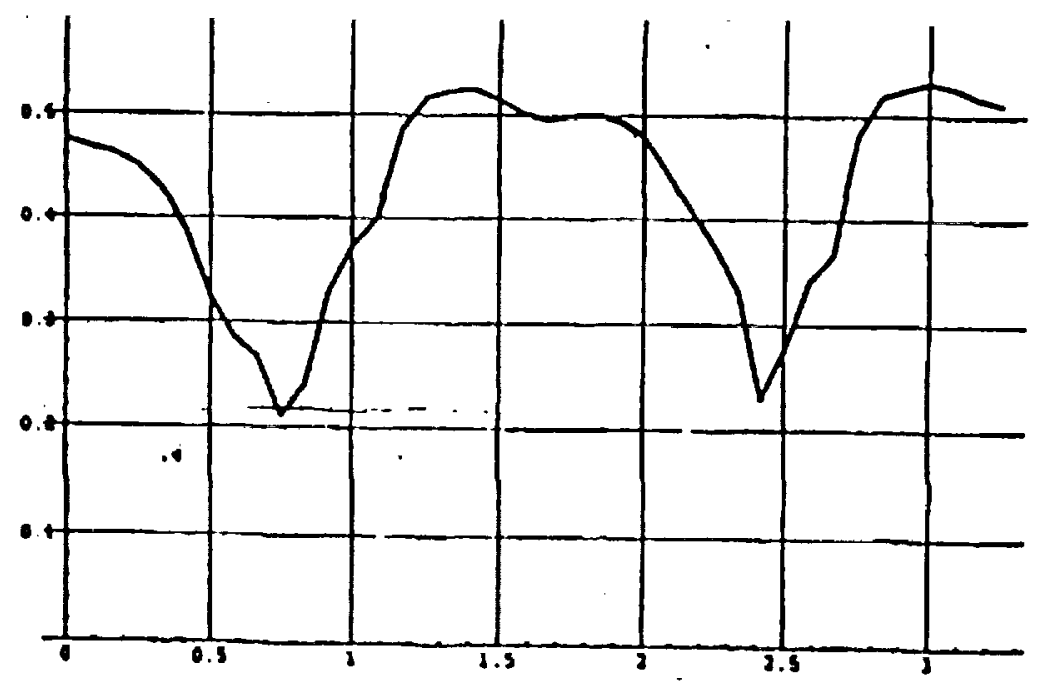

Time Around Orbit (hrs)

Fig. 6 Thrust for the $7 \cdot \mathrm{km}$ long tether at $5 \mathrm{~kW}$.

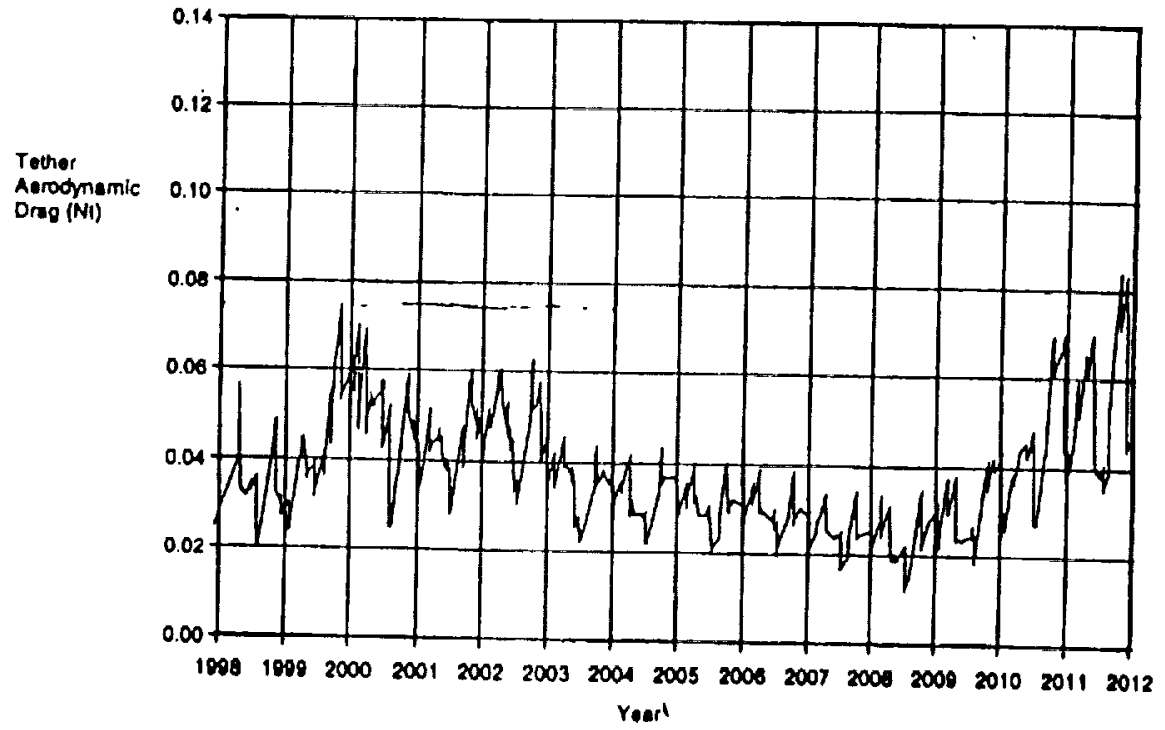

Fig. 7 Ribbon tether uerodynamic drag.

must be long enough to accommodate the lowest anticipated electron densities, but only as much of it as needed will actually carry currenl.

\section{Deployer Strategies}

\section{Tether Deployment}

We have considered alternatives in deployer design, grouped into three general types: 1) expendable, 2) payout and retrieve, and 3) up and down.

\section{Expendable}

Expendable tether systems, in which the tether is simply cut after use and allowed to naturally drift away from the platform to even. lually fall and burn in the upper atmosphere, are generally simpler, lighter, and cheaper to build. The obvious downside of expendable lethers is that they are good for a single use and must be discarded and replaced in cases where damage or environmental factors may unly temporarily require its removal. If a deployed tether is judged incompatible with the arrival of any spacecraft, then many expendable cethers would be discarded per year.

Retrievable Deployment Martin Marie"ll

The principal example of a deployer designed to tetrieve a tether, as well as paying it out, is the design by the Martin Company for the TSSI and TSS-IR tethered satellite experiments. ${ }^{4}$ Th 1 s deployer was heavy $(2000 \mathrm{~kg})$ with a large deployment boom. Part of the reason for this weight was undoubtedly its intended generality. It was built 10 accommodate tethers up $10100 \mathrm{~km}$ long with end weights of up $10500 \mathrm{~kg}$ mass. This deployer was flown twice and suffered from problems of tether snagging and breakage.

Payout is similar for both expendable and retrievable tethers, but retrieval docs lead to polentially severe problems of control. Any lateral motion is amplified by the conservation of angular momen. tum as the tether's moment arm is shortened. This leads to insta bilities, which require active control strategies. This is most acute in the late stages of retrieval, where each meter-of-length reduc. tion leads to an increasingly large fractional change in the reduc- 


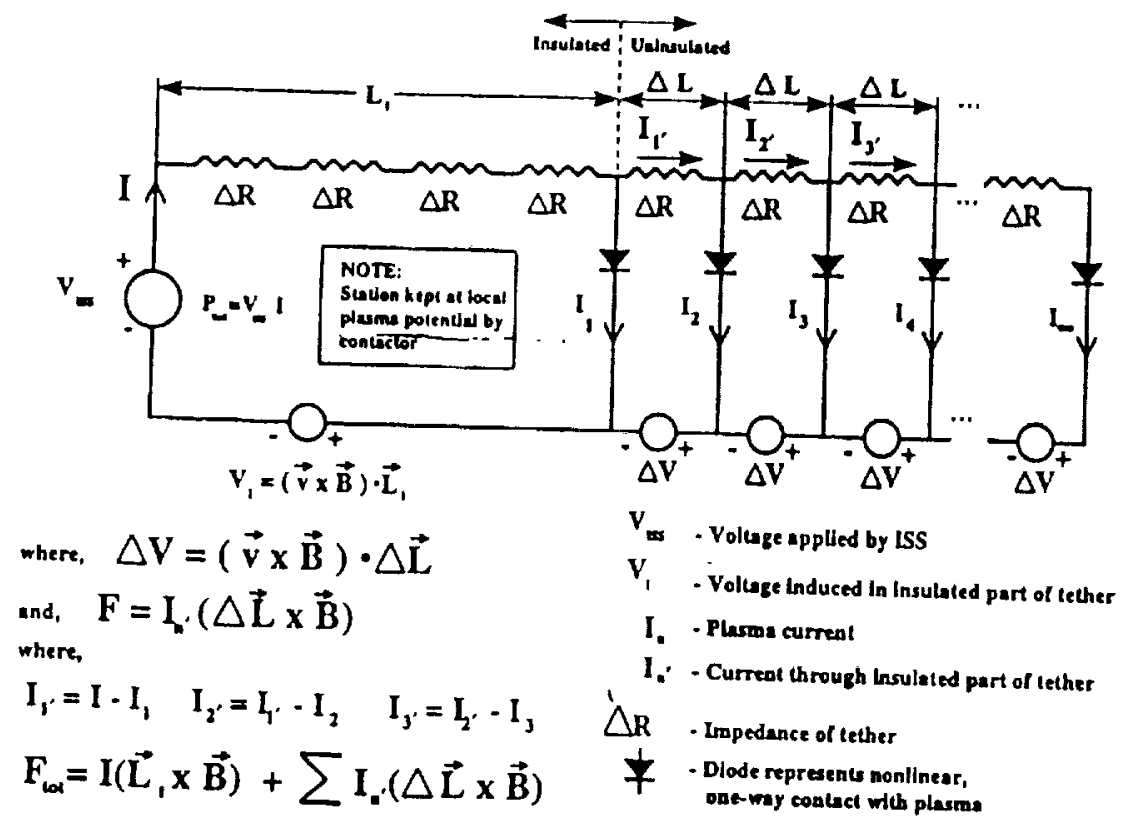

Fig. 8 Bure-tether currents and forces.

To rise tether.

Delach reboost power elecurode.

Aluch and-mass clectron-collector.

Deploy upper masscallector.

Raise lether with gripper wheels.

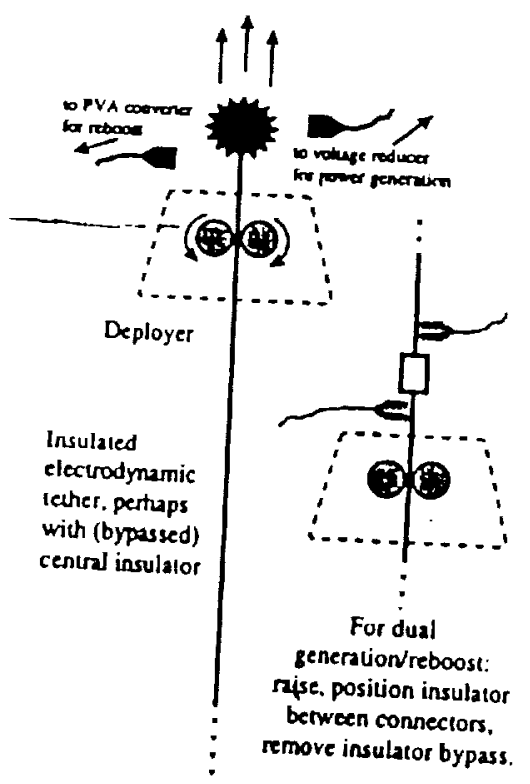

g. 9 Pass-Through electrodynamic tether.

Solving these retrieval problems will be necessary in significant permanent presence in the magnetosphere periods. The tether itself may be a heavy and expen1 intended for use over periods much longer than a cle, as it would be over the life of a space station many service vehicles per year.

\section{deployment}

trieval problems are likely to occur in the later stages These and other considerations led us to consider I rather different approach to retrieval: rather than her back on its spool, let it pass straight on through effectively redeploy on the oppositc side (Fig. 9).

l lethers, which extend in both directions (nadir and e platform, have been considered. These allow easy switching between power generation and reboost functions (functional reversal) and have minimal effect on the platform's orbit or center of mass (CM). Although it can provide either reboost or power, power generation is likely to be a secondary function, supplementing solar power only in unusual or emergency circumstances because it operates by draining orbital energy.

Advantages of pass-through deployment and retrieval include possible functional reversal and snag-free redeployment.

The general requirements for pass-through tether retrieval are as follows: I) electrical disconnection from platform power sources or loads, 2) altachment of an alternative endmass for stability/contro or an electron collector for funclional reversal in place of an end mass power source or load, 3) a mechanical means of gripping and moving the tether at any point along its length, and 4) a path through the platform to the other side with sufficient angular clearance for both deployment and retrieval.

Other specific advantages and disadvantages depend upon whether the intent is to redeploy fully the tether on the opposite This choice through extensionds upon the motivation for retrieval. The pass-

\section{Full Pass Through}

If the motivation is either to 1) remove the tether from potential interference with a service vehicle approaching from beneath, 2) shift the platform's CM, or 3) repair damage at or near the tether's outer end, then a nonrewinding retrieval must pass the full tether length through or past the deployer and its platform. The process is
illustrated in Fig. 10 .

A full pass through has the following advantages: i) fine tuning of platform $\mathrm{CM}$ and torque equilibrium angle (TEA); 2) control by rolating tether boom about the $y$ axis; 3) freedom from conflic with approaching vehicles on the original side; 4) ability to access a damaged region anywhere along its length; 5 ) full funclionality for both power generation and reboost; 6) electrical reconnection at original site of the electron collector; and 7) if configuration acceptwithout electrical hazard.

Disadvantages include the following: 1) disconnection of original electron collector; 2) if bare tether originally used for electron collection, then rewinding or separate retrieval needed to resolve 4) clectrodynamic 3 ) $\mathrm{CM}$ and TEA shift possibly significant, and or TEA is motivation (because consion likely if adjusiment of CM platform). 


\section{Full reboost}

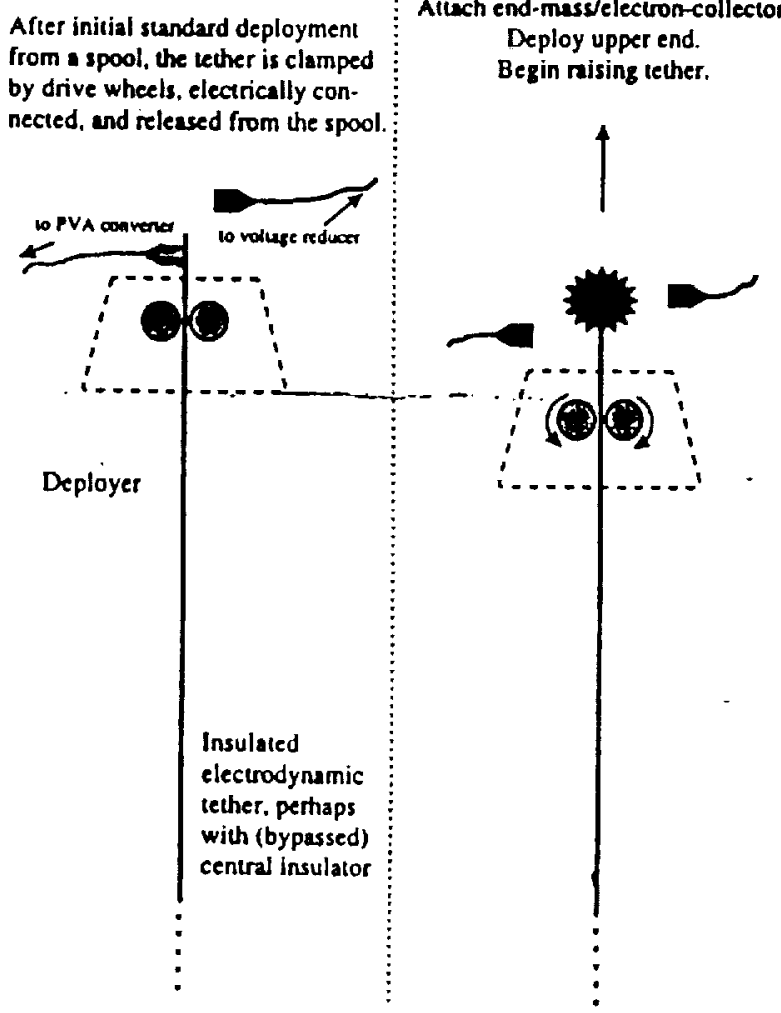

\section{Full generation}

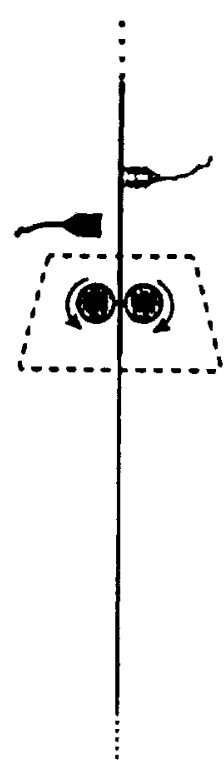

Dormant

bare tecther

electron collector

Fig. 10 Fully bidirectional tether concept.

Again, this design neither helps nor worsens the retrieval control problem.

\section{Alternative Power Sources and Locations}

The current design of the ISS was used to delermine suitable locations for the physical and electrical attachment of a deployer and its power supplies. The full-up station carries four pairs of photovoltaic arrays capable of generating $20 \mathrm{~kW}$ each. Of this $80 \mathrm{~kW}$ $54 \mathrm{~kW}$ are intended for housekeeping functions. I is not expected that the $5 \mathrm{~kW}$ planned for reboost would overburden the remaining user allocation. The station's batteries will support the same power availability as sunlight operation.

The tether current is limited by the excess electron rejection calpacity of the currently designed plasma contactor. This contactor has a nominal design rating of $10 \mathrm{~A}$, with normal operation requiring 2-3 A to maintain the station to within $40 \mathrm{~V}$ of ambient plasma potential. This should readily permit a $5 \mathrm{~A}$ default allocation to tether reboost power. Modest short-term overcurtent demands are not harmful, other than somewhat increasing the normal depletion of the hollow cathode's xenon supply. Nevertheless, if a stronger lether thrust is to be used, to totally compensate drag with a shorter duty cycle or even gain altitude, this contactor should be replaced by one with larger design capacity.

Tether reboost operation can be ireated as a low-priority resource demand, so long as long-term planned duty cycle requirements are met on average. Thus, tether power can be cut to accommodate peak user demand times with no effect other than a change in net aerodynamic drag forces averaged over time. The accelcration induced by tether thust is approximately $0.4 \mathrm{~N} / 400,000$ $\mathrm{kg}=10^{-\kappa} \mathrm{m} / \mathrm{s}^{2} \approx 0.1 \mu \mathrm{g}$, which is low for even the station's best $\mu-$ gravity environments. This tether thrust will normally improve the $\mu$-gravity environment by canceling the comparable deceleration from aerodynamic drag. although it is possible that extremely sensitive payloads might be affected by rapid changes in this range.

Likely physical attachment locations for a tether deployer are on the SO truss, the ZI truss, or a direct mounting on Node I. Node II considered a good choice because it is close to the station's CM, al.

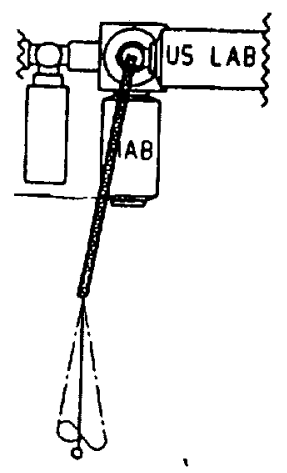

Fig. 11 Tether attachment.

though a truss location may be preferable if any of the pass-through deployment options presented in this paper are adopted. To avoid mechanical interference, the tether must honor an envelope to ensure clearance of all ISS hardware under normal, abnormal, and abort conditions. A 10-deg cone of operational clearance should suffice because tether libration must be controlled to less than that value. In principle, one may altach a small truss at any convenient location, as long as it extends to a position below station where the deployer boom can give a tether alignment that exerts the desired torque levels (or lack thereof) on the station. For the most flexible control of the tether's applied torque and its consequent effect on the station's torque equilibrium angle, the tether's force vector should pass reasonably near the $\mathrm{CM}$, but guided by a boom with freedom of rotation about a $y$ axis, as shown in Fig. 11 .

Electrical power can be oblained by connection to the station's main bus switching unit, where power has been conditioned to 160 VDC (Fig. 12). The tether's own power supply will raise this 160 VDC to the 1500 VDC required to overcome the tether's motioninduced voltage and drive as much as $5 \mathrm{~A}$ of downward current. This power supply includes an inverter, transformer, rectifier, filter, and regulator as shown in Fig. 13. Because of its high voltage output, the power supply should be located close to the deployer rather than to its power source. 


\section{Reboost Options}

The primary benefits of the electrodynamic tether system are the savings to the ISS from reduced propellant mass requirements and the extension of time between disruptions from planned propellant proximately three one-year periods following altitude profile for apwere analyzed following assembly completc were analyzed assuming an operational electrodynamic tether sys from Fig lime periods chosen were 2003, 2006, and 2009, which, from Fig. 1, are collectively representative of the ISS orbital decay profile. The assumed electrodynamic tether force ranged from 0.43 10 $0.7 \mathrm{~N}$, with a duty cycle ranging from $251050 \%$.

Gauss's form of Lagrange's variational equations can be used to describe the decay of the ISS orbit.s For a circular orbit the mean orbital angular rate is given by

$$
n=\sqrt{\mu / a^{3}}
$$

The lime rate of change in the semimajor axis $a$ is described by

$$
\dot{a}=2 T / n
$$

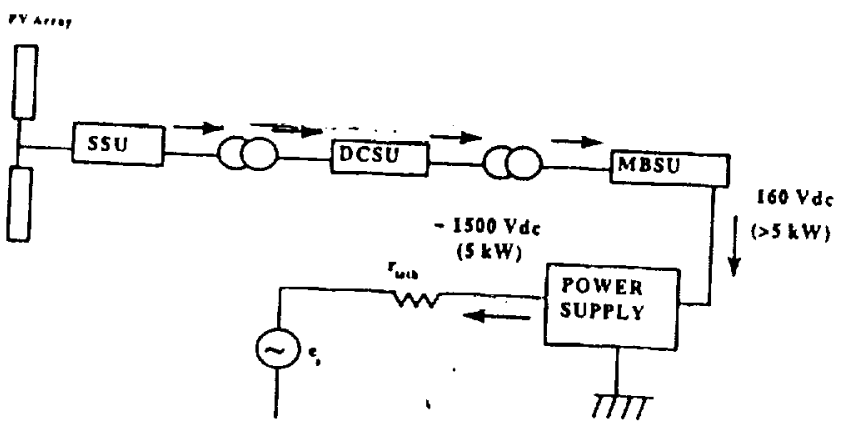

Fig. 12 Tether power connection: SSU, sequential shunt unit; DCSU direct current switching unit; MBSU, main bus switching unit; $r_{\text {letli, }}$ lether's electrical resistance; and $e_{3}$, tether's Induced voltage.

Asoumptions

- Tether requires $5 \mathrm{~kW}$ trom ISS

- Tether requires 1500 Vdc

- ISS provides $160 \mathrm{Vdc}$

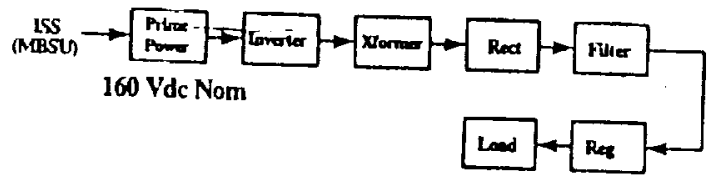

$P w r=5 \mathrm{kWe}$

Eout -1500 Vmax

Fig. 13 Power supply for tether.
The tangential perturbation acceleration $T$

$$
T=-\frac{1}{2} \rho v^{2} C_{d} A / m+F_{1} / m
$$

possesses two components: the first from aerodynamic drag, as already described, and the second from the electrodynamic tether thrust acting on the ISS mass. Equation (5) is separable and can be integrated in closed form by assuming constant values for the slowly varying parameters. This restricts the validity of the for the to small time intervals or equivalently small altilude changes.

We use the subscript $i$ to denote an initial configuration at a start. ing time $t_{i}$ and semimajor axis $a_{i}$, and the subscript $f$ to denote the value at some future time $t_{f}$, where $t_{f}>t_{i}$ and the semimajor axis has evolved to $a_{f}$. Integrating Eq. (5) from $t_{i}$ to $t_{f}$, the new semimajor axis $a_{\text {, can be expressed as }}$

$$
a_{f}= \begin{cases}\alpha \sigma & \text { for } a_{i}<\alpha \\ \alpha / \sigma & \text { for } \quad a_{i}<\alpha \\ \alpha & \text { for } \quad a_{i}=\alpha\end{cases}
$$

where

$$
\alpha \equiv \rho C_{d} A \mu / 2 F_{1}
$$

and

$$
\sigma \equiv[(1+\gamma) /(1-\gamma)]^{2}
$$

with

$$
r=\left[\frac{\left( \pm \sqrt{a_{i}}-\sqrt{\alpha}\right)^{2}}{ \pm a_{i} \mp \alpha}\right] \exp \left\{ \pm\left[\left(2 F_{1} / m\right) \sqrt{\alpha / \mu}\right]\left(t_{j}-l_{i}\right)\right\}
$$

The upper sign is used over the interval of integration when $a>\alpha$ and the lower sign for $a<\alpha$. Here, $\alpha$ can be understood as the semimajor axis multiplied by the ratio of the aerodynamic drag to the tether force. When these opposing forces balance, then

$$
a_{f}=\alpha=a_{i}
$$

When drag and tether forces are unbalanced, the semimajor axis will deviate from $a_{i}$. As expected, if the tether force dominates drag and $a_{i}>\alpha$, then the semimajor axis will increase, and the station will gain altilude. If drag dominates and $a_{i}<\alpha$, then the station will As fall.

As mentioned earlier, this solution becomes less accurate for large altitude changes, which necessitates a piecewise approach to its the variotion. By evaluating this expression for small time intervals. the variation in those parameters held constant during the analysis can be accommodated by iteratively specifying updated values for each subsequent evaluation period.

This approach was used to construct a modificd reboost profile for the time periods mentioned earlier (Fig. 14). The electrodynamic

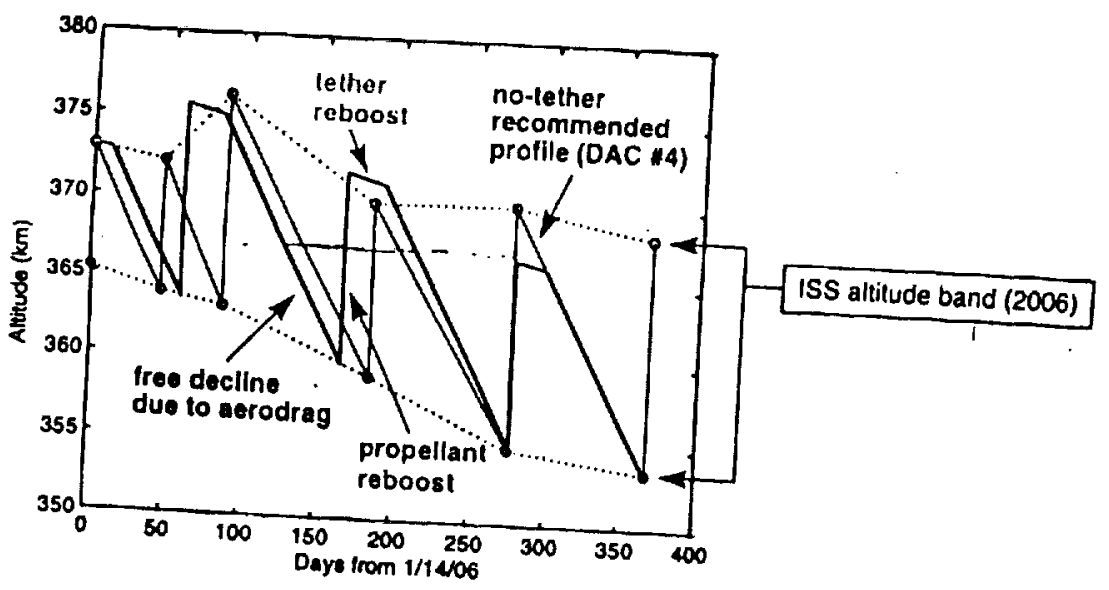

Fig. 14 Tether reboost profile- $5 \mathrm{~kW}$. 


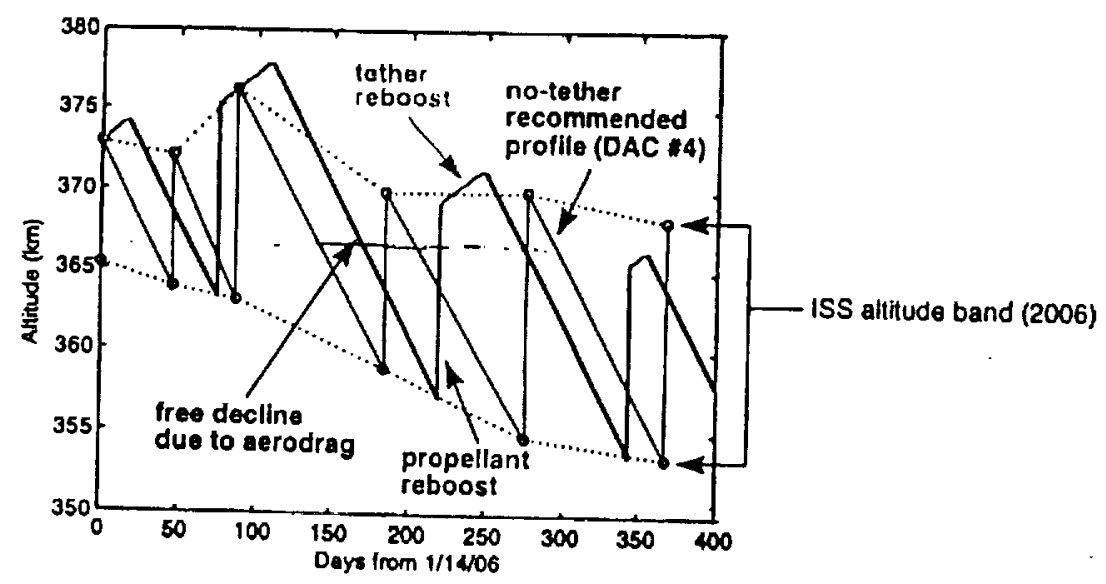

Fig. IS Tether reboost profile-10 kW.

tether system was assumed to operate at $5 \mathrm{~kW}$, which provides a propulsive force of $0.43 \mathrm{~N}$, slightly less than the aerodynamic drag force illustrated in Fig. 3. Tether reboost, free decay, and propellant reboost correspond in Fig. 14 to changes in slope between successive end of free dectart of tether reboost and the end of free decay was iteratively determined. Propellant reboosi band (as defined by boundaries of the altitude band (as defined by Fig. 1), subject to the constraint that tether reboost occurs during $25 \%$ of this interval (i.e., $25 \%$ duty cycle). Propellant reboost maneuvers are very short compared to orbilal decay times and were assumed to occur instantaneously.

Altilude change caused by propellanl reboost was determined us. ing the familiar rocket equation and Hohmann transfer relationships. At the end of the free decay, the semimajor axis $a_{1}$ and the circular orbital velocity $v_{l}=\sqrt{ }\left(\mu / a_{1}\right)$ are known. As usual, the two im. pulsive maneuvers are assumed to occur instantaneously. The first the orbit by raising perigee aneas the second maneuver circularizes the orbit by raising perigee an equivalent distance. Theoretically, the orbital period which is neen these two maneuvers is one-half the velocity incremich is negligible on the scale of Fig. 14. The total of propellant rocket equation as

$$
\Delta u=-g_{01} I_{s p} \log \left(1-\Delta m / m_{r}\right)
$$

using gravitational acceleration $g_{i}=9.81 \mathrm{~m} / \mathrm{s}^{2}$, specific impulse $f_{\mathrm{sp}}=300 \mathrm{~s}$, and reboost mass $m_{r}$, which exceeds $m$ by the mass of the resupply vehicle. Denoting the velocity on the raised circular orbil by $v_{2}$, the velocity ratio $\eta=v_{2} / v_{1}$ can be determined from the Hohmann transfer relationships as

$$
\Delta u / \nu_{1}+(1-\eta)\left[1-(1-\eta) \sqrt{2 /\left(1+\eta^{2}\right)}\right]=0
$$

Solving numerically for $\eta$, the velocity on the raised circular orbit is then determined from $v_{2}=\eta v_{1}$ with the corresponding semimajor axis $a_{2}=\mu / u_{2}^{2}$ and, hence, altitude $h_{2}=a_{2}-R_{c}$.

The effects on the reboost profile of operating the electrodynamic tether at higher power can be seen in Fig. 15. This figure illustrates the savings of two propellant reboost flights (or equivalently $2000 \mathrm{~kg}$ of propellant) to the ISS for the year 2006. The electrodynanic tether sive force of $0.7 \mathrm{~N}$ operating at $10 \mathrm{~kW}$, which provides a propuldrag force illustrated in somewhat greater than the acrodynamic was again $25 \%$. The higher. 3 . The duty cycle for the tether reboost ply flight by over two months. Clearly apidly if the duty cycle can be increased savings here will increasc

$$
\text { A similar analysis was performed for. }
$$

A similar analysis was performed for the years 2003 and 2000 . Figure 16 summarizes these results by illustrating the annual propelBased upon the range of the electrodynamic tether reboost system. boost system can reduce the numbers considered here, the tether reby one to four annually.

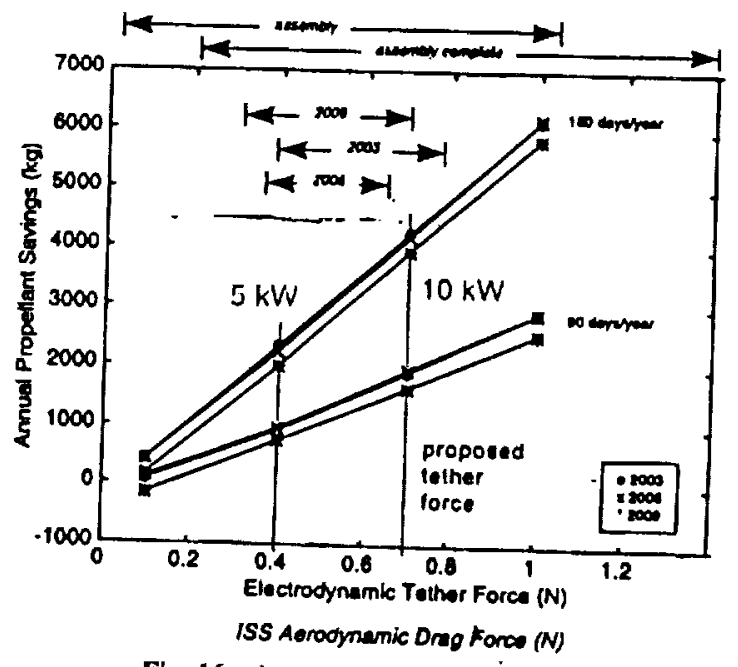

Fig. 16 Annual propellant savings.

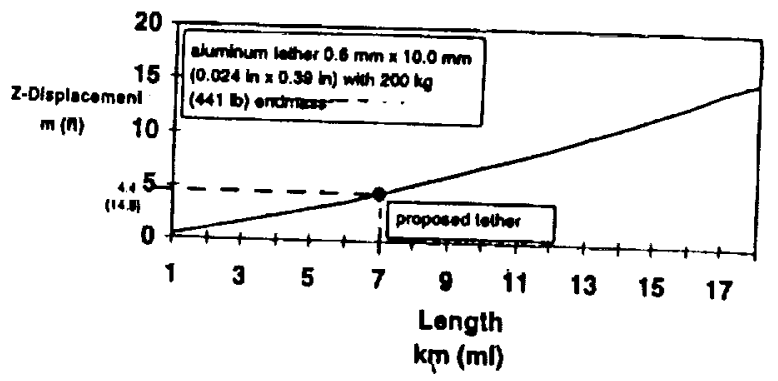

Fig. 17 Displacement of ISS CM by lether.

\section{Impacts to ISS}

The use of an electrodynamic tether to provide reboost to the in this section.

\section{Efrects on $\mathrm{CM}$ and $\mu$ Gravity}

The proposed tether does have a significant impact on the $\mathrm{Z}$ com. ponent of the station's CM. The tether itself would impact the CM because of its long moment arm. Figure 17 shows that a $7-\mathrm{km}$ tether of the proposed lype, with a $200-\mathrm{kg}$ end mass, would lower the station's CM by about $4.5 \mathrm{~m}$. The prepared tether configuration would lower the projected $\mu$-gravity contours from those currently planned without a tether to those shown in the bottom part of Fig. 18 . Using the tether, the region of best $\mu$ gravity has been shifted from the top of the U.S. Iab to the boltom, with a similar shift-and possibly even an improvement-for the European and Japanese labs. If this shift is deemed undesirable, $i$ could be reduced by maneuvering the 

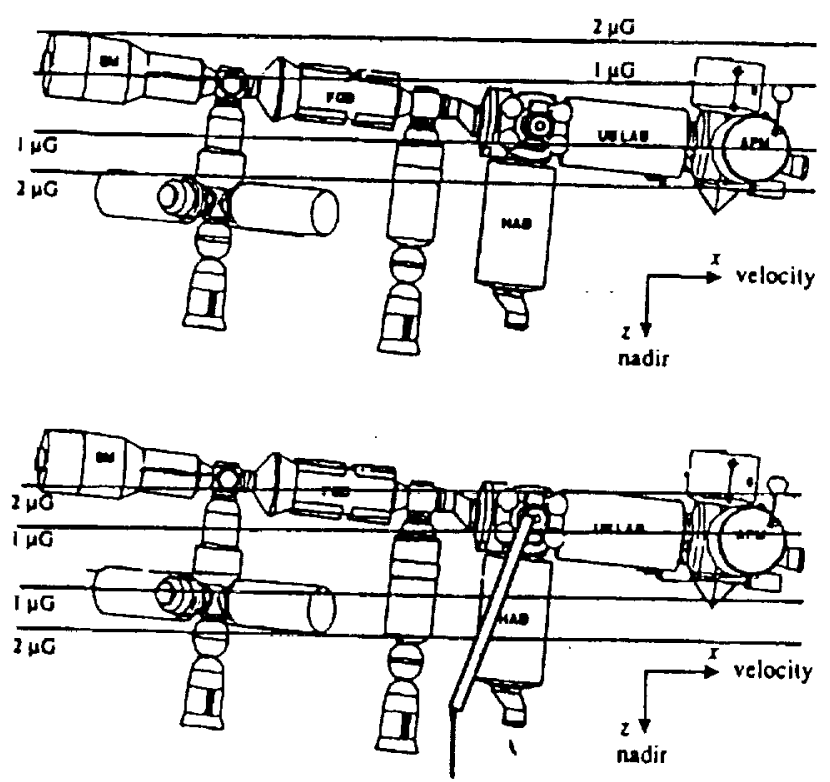
Fig. 18 Microgravity environment: top, without tether, and botlum,
with tether.

tether boom, shown in Fig. 11, to adjust the station's TEA so as (1) lower its leading edge.

The effecl of the tether's deployer on the $X$ componem of the slation's CM is negligible because even a $500-\mathrm{kg}$ deployer located $20 \mathrm{~m}$ forward of the $\mathrm{CM}$ would move the $\mathrm{CM}$ forward by only $2.5 \mathrm{~cm}$. $X$-axis displacements are thus of less concern than vertical ( $:$ axis) displacements because of the tether mount's small moment arm and the low-gravily gradient force.

\section{Power Demands upon ISS}

The tether requirement for $5 \mathrm{~kW}$ (or $10 \mathrm{~kW}$ ) of power from the 80 $\mathrm{kW}$ available from the generating sources does not appear excessive during the daylight hours. Extracting this level of power from the bat. teries during night operation may not be satisfied at all times. Under these conditions, the tether power and the reboost thrust would be reduced accordingly.

\section{Retrieval or Jettison of Telher-Normal Conditions}

The tether system for reboost is intended to be used during qui. cscent periods because it would tend to nullify the station drag and lhus improve rather than degrade the quality of microgravity. At limes when visiting spacecraft come to the ISS, the extended lether might interfere with their docking procedure. Under these circum. stances, the tether would need to be retrieved, or cul and discarded, prior to the spacecraft's visit. It is expected that spacecraft will be risiting on a fairly regular basis-six flights at least for orbiter, four 10 six flights for Progress $M$, and flights by other spacecraft-so cutting and discarding the tether may become a cost issue. Retrieval of the tether appears a candidate solution but raises control issues. particularly when the tether length is short (less than approxiniately $1 \mathrm{~km}$ ). Retrieval systems inherently carry higher initial costs than expendable systems. Further analysis and design will be needed to
rcsolve these issues.

\section{Maneuverable Boom to Guide Tether}

A buom may be used for tether deployment. The boom can move the tether away from other elements of the ISS and place the $x$ component of the tether c.g. near the c.g. of the Station. Furthermore, a boom may be used to optimize the station's torque equilibrium angle to minimize the frequency of rocket firings to desaturate the control moment gyros and thus save even more propellant.

\section{ISS Survivability and Cost Savings}

Over the past several years, the Russian resupply vehicle Progress $\Lambda$ has proven itself to be a highly reliable means of delivering life

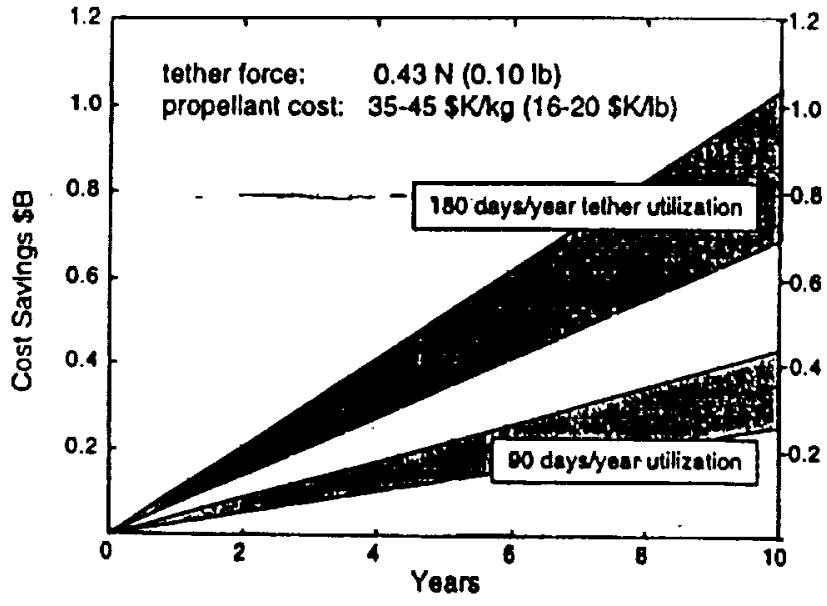

Fig. 19 Cumulative cosl savings.

support materials and reboost capability to low Earth orbit. Of the propellant reboost options considered for the ISS, only the Progress $M$ is operational. The Soyuz launch vehicle is used to insert Progress $M$ into low Earth orbit at a cost of approximately $\$ 15-25$ million (Ref. 6). The propellant cost is an additional $\$ 20$ million for a total cost of between $\$ 35-45$ million. Commercial sources indicate that this value may be as high as $\$ 65$ million. The $\$ 35-45$ thousand/kg estimate of Progress $M$ propellant launch cost (i.e., the lower cost number) is used in this paper to ensure more conservative estimates of cost saving.

Propellant savings provided to the ISS are illustrated in Fig. 16. These can be converted to dollar savings using the $\$ 35$ thousand $/ \mathrm{kg}$ launch estimate for propellant. Figure 19 illustrates the results of this conversion expressed as cumulative cost savings for the ISS over 10 years of operation. The two shaded regions correspond to 25 and $50 \%$ duty cycles of tether reboost operation for a tether force of $0.43 \mathrm{~N}(5 \mathrm{~kW})$. The widening of these regions with time results from the differences in the computed annual propellant savings between the three different one-year periods considered. Al the higher duty cycle a savings in excess of one billion dollars over the operational life of the ISS is possible by using an electrodynamic tether to supplement propellant usage. Al the higher reboost value of $0.7 \mathrm{~N}(10 \mathrm{~kW})$, the savings are approximately twice as
nuch.

These estimate savings may pale in significance, however, in com. parison to the role of a tether as a backup system to ensure ISS survival in case propellant resupply flights should be internupted for any extended period. During an extended period of isolation, a tether could provide service for an extended duration with a much higher duty cycle than has been considered here, even if the tether were judged incompatible with safety requirements for vehicle docking and even if only a nonretrievable tether were available.

\section{Conclusions}

Multiple benefits are accrued by the use of a propellantless system to reboost the ISS. Because the ISS is designed as a research and test facility to fly in an inherently unstable orbit to ensure reachability for over 10 years, means of providing reboost is a critical concern. The electrodynamic tether-based reboost system could satisfy some of the total reboost needs of the ISS, resulting in a reduction in flights that deliver propellant. The higher the usage of the electrodynamic tether, the larger the cost savings because of the need for fewer propellant resupply flights. Use of this method of reboost would provide for additional quiescent days as the system would have no major impact on the microgravity environment and under certain conditions could improve the microgravity environment. In addilion. a maneuverable boom could provide some variation/control in the ISS torque equilibrium attitude. In addition to economic considerations, an electrodynamic tether offers a practicle backup system to ensure ISS survival in the event of an (otherwise) catastrophic
delay in propellant delivery. 


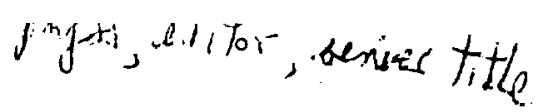

CSC Computse Sciences Corporation

COTR Contracting Officer's Technical Representative

ar 2 no anthors

ist 3 No arthors

Det 5 Do not have poges

jet 6 Tsokuwitz - edifor

This is not a part of a "Sories"

pages - not aumitable 